
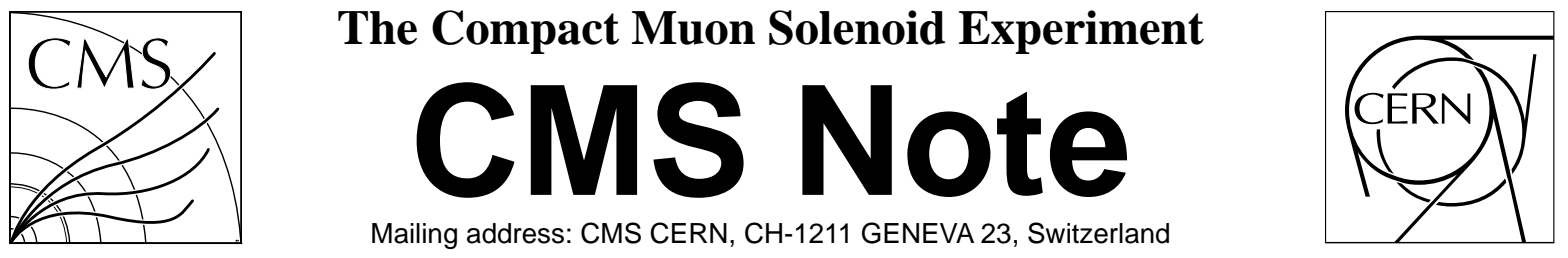

1 Oct. 2003

\title{
The Efficiency of the CMS Level-1 Trigger for Supersymmetric Events
}

János Erö, Massimiliano Fierro, Anna Jeitler, Norbert Neumeister, Paul Porth, Herbert Rohringer, Lali Rurua, Hannes Sakulin, Anton Taurok, Claudia-Elisabeth Wulz

Institute for High Energy Physics of the Austrian Academy of Sciences, Vienna, Austria

László Boldizsár, Pál Hidas

RMKI Budapest, Hungary

\begin{abstract}
Results on the physics performance of the CMS Level-1 Trigger for supersymmetric events at an LHC luminosity of $2 \times 10^{33} \mathrm{~cm}^{-2} \mathrm{~s}^{-1}$ are given. Energy and momentum trigger thresholds have been chosen to yield a maximum Level-1 output rate of $50 \mathrm{kHz}$, within a safety factor of three. The Level-1 trigger efficiencies for the majority of the studied channels are found to be greater than $90 \%$, providing a good basis for the High Level Trigger, where more stringent conditions are applied than at Level-1. Reasons for occasional lower efficiencies are given.
\end{abstract}




\section{Introduction}

The Large Hadron Collider (LHC), foreseen to begin operation in 2007, is presently under construction at CERN, the European Organization for Particle Physics in Geneva. The initial luminosity for proton-proton collisions at $14 \mathrm{TeV}$ center-of-mass energy is expected to be $2 \times 10^{33} \mathrm{~cm}^{-2} \mathrm{~s}^{-1}$. At this luminosity, also denominated as low luminosity, an average of 3.5 proton-proton interactions will occur every $25 \mathrm{~ns}$, corresponding to a bunch crossing frequency of $40 \mathrm{MHz}$. In practice the fraction of filled bunches in the LHC ring is 2808 out of a total of 3564 , therefore the average bunch crossing rate is $31.5 \mathrm{MHz}$. This leads to an interaction rate of more than $100 \mathrm{MHz}$. Since it is impossible to store and process the large amount of data associated with the resulting high number of events, a drastic rate reduction has to be achieved. This task is performed by the trigger system, which is the start of the physics event selection process.

CMS (Compact Muon Solenoid), one of two multi-purpose experiments at the LHC, has chosen to reduce the input rate in two steps called the Level-1 (L1) Trigger [1] and the High Level Trigger (HLT) [2] respectively. A decision to retain an event for further consideration has to be made by the L1 Trigger for each bunch crossing. This decision is based on an event's suitability for inclusion in one of the various data streams to be used for analysis. The data sets to be taken are determined by CMS physics priorities. They include data sets triggered with single leptons and dileptons, mainly for Higgs boson and other discovery searches as well as for B- and heavy ion physics. Data sets triggered with leptons plus jets are important for supersymmetry (SUSY) and top physics, and inclusive electron data sets are useful for calorimeter calibrations. In addition, other samples are necessary for measuring event selection efficiencies and studying backgrounds. The trigger has to select these samples in real time along with the main data samples.

The L1 Trigger has to take the decision whether to accept or to reject an event for each bunch crossing with negligible deadtime. The crossing interval of $25 \mathrm{~ns}$ is too short to process the complete trigger information, therefore a pipelined system that stores the data during a latency time of $3.2 \mu \mathrm{s}$ has been conceived. The rate reduction capability is designed to be at least a factor of $10^{6}$ for the combined L1 and HLT, as the highest rate that can be archived by the on-line computer farm is of the order of $100 \mathrm{~Hz}$. The maximum bandwidth allocated to the L1 Trigger is $100 \mathrm{kHz}$, in accordance with the input capacity limit of the HLT processor farm. However, at the startup of LHC, it will be reduced to $50 \mathrm{kHz}$ for operational reasons.

The trigger efficiencies for SUSY events calculated in this paper are those at the end of the Level-1 chain, i.e. at the output of the Global Trigger. The CMS Global Trigger takes the decision to accept or reject an event at L1, based on input from the Global Calorimeter and Global Muon Triggers. The latter two process information from the Regional Trigger of the calorimeters and the muon system. The CMS Muon Trigger is special in the sense that both the precision tracking chambers, made up of drift tubes in the barrel and cathode strip chambers in the endcaps, and dedicated resistive plate trigger chambers take part in the trigger. The calorimeter and muon Local Trigger at the bottom end of the trigger chain is based on energy depositions in calorimeter trigger towers and track segments in muon chambers respectively.

In the following chapters the CMS Level-1 Trigger, the simulated physics channels as well as the detector and trigger simulation are described, followed by a definition of the applied trigger scenario. Finally the results on trigger efficiencies obtained with the used trigger menu are presented and discussed.

\section{The CMS Level-1 Trigger}

The CMS Level-1 Trigger operates on the basis of trigger objects delivered by the calorimeter and muon trigger systems. These objects are candidate electrons or photons, muons, jets, $\tau$-jets as well as total and missing transverse energies and jet multiplicities. Neither the silicon inner tracker nor the pixel vertex detector takes part in the Level-1 Trigger.

The CMS calorimeter consists of a homogeneous electromagnetic part made of lead tungstate crystals and a hadronic part made of brass and scintillator samples in the barrel and endcap regions and quartz fibres embedded in an absorber matrix in the forward region. The first step of the Calorimeter Trigger consists of summing the energies measured in cells within electromagnetic and hadronic trigger towers. In the region up to $|\eta| \approx 2$ 
each trigger tower has a pseudorapidity-azimuth $(\eta-\phi)$ coverage of $0.087 \times 0.087$ rad. Beyond that boundary the towers are larger. The Regional Calorimeter Trigger [1,3] then determines candidate electrons, jets and $\tau$-jets as well as transverse energy sums. The candidates found in a region of $\Delta \eta \times \Delta \phi=3.0 \times 0.7 \mathrm{rad}$, which corresponds to one trigger crate in hardware, are then ranked according to transverse energy $\left(E_{T}\right)$ and quality. The four with the highest rank in each region are transmitted to the Global Calorimeter Trigger [4], which in turn determines the four highest rank calorimeter trigger objects across the whole CMS detector.

Electrons and photons are indistinguishable at the level of the L1 Trigger due to the absence of tracker information. The electron trigger algorithm, applied across the entire $(\eta, \phi)$-plane, starts by determining the tower with the largest energy deposit. The energy of the tower with the next-highest deposition in one of the four broad side neighbours is then added. Isolated and non-isolated electrons are determined by the trigger. A non-isolated electron requires passing of two shower profile vetoes, the first one based on a fine-grain crystal energy profile reflecting the lateral extension of a shower, the second one on the ratio of the deposited energies in the hadronic and in the electromagnetic sections. A typical maximal value of 5\% is allowed for the latter. An isolated electron candidate has to pass the previous vetoes for all eight neighbouring towers. In addition, at least one quiet corner made out of four groups of five towers surrounding the hit tower is required.

Jets are found by a technique using a sliding window of $3 \times 3$ calorimeter regions corresponding to $12 \times 12$ trigger towers in the barrel and endcaps and covering a region of $\Delta \eta \times \Delta \phi=1.04 \times 1.04 \mathrm{rad}$. In the forward hadronic calorimeters $3 \times 3$ larger trigger towers are grouped together. The window is shifted by one trigger tower at a time. The transverse energy of the region made up by the $4 \times 4$ central trigger towers is required to be higher than the eight neighbour region values. It is also required to be larger than $1 \mathrm{GeV}$ to suppress soft spurious contributions.

$\tau$-jets from single and three-prong $\tau$-decays are required to have a narrower transverse energy distribution than ordinary quark/gluon jets. A jet is declared a $\tau$-jet if none of the nine calorimeter trigger regions has a $\tau$-veto bit on. Such a veto bit is set unless the pattern of active towers corresponds to at most $2 \times 2$ contiguous trigger towers within a $4 \times 4$ tower region.

In addition to electrons and jets the Regional Calorimeter Trigger also delivers transverse energy sums and jet multiplicities to the Global Calorimeter Trigger. The latter determines the total transverse energy, the magnitude and direction of the missing transverse energy computed from the tower energy measurements in the two coordinates transverse to the beam within $|\eta|<5$, and twelve jet counts for different energy thresholds and optionally also different $\eta$-regions. The jet counts were not used in this study, but may be useful to trigger on events with a large number of jets, such as gluino or squark decays. The Global Calorimeter Trigger also delivers $H_{T}$, the scalar transverse energy sum of all jets above a programmable threshold - $10 \mathrm{GeV}$ for this study. It is less sensitive to noise and pile-up than the total transverse energy and will, just like the jet counts, be particularly useful if events have many but relatively low energy jets. Such events may actually fail the jet triggers that have to use high thresholds to achieve an acceptable trigger rate.

All three CMS muon subsystems take part in the trigger and can deliver information on transverse momentum $\left(p_{T}\right)$ and location of muon candidates. The tracking chambers in the barrel and in the forward regions are equipped with electronics suitable for triggering. Precise timing information is delivered both by the drift tube (DT) and cathode strip chambers (CSC). The resistive plate chambers (RPC) mounted on the tracking chambers are dedicated trigger chambers.

The barrel DT chambers have a special Bunch and Track Identifier logic [5] to find track segments from coincidences of aligned hits in four layers of one drift tube superlayer. All DT chambers have two superlayers to measure azimuth. The segments from these two layers are combined by a track correlator, which works on the basis of the measured angular coordinates. The chambers in the three innermost muon stations have a third superlayer to determine pseudorapidity. Its wires are orthogonal to the wires of the two $\phi$-superlayers. The fourth outermost muon station of CMS has only two $\phi$-superlayers.

The trigger electronics of the endcap CSC chambers forms Local Charged Tracks (LCT) from the cathode strip measurements [6]. These are combined with information from anode wires for bunch crossing identification. The highest- $p_{T}$ DT and CSC track segments are sent to the respective regional triggers called Track Finders [7, 8]. 
They form muon candidates out of the track segments using extrapolation and pattern matching methods. The RPC's deliver hit patterns. Muon candidates are found by comparing the found patterns with precalculated ones [9].

Since three different muon subsystems take part in the trigger, CMS needs an entity to determine unique muon candidates. This entity is the Global Muon Trigger [10]. It sorts the DT, CSC and RPC candidates and tries to match them. It achieves an increased efficiency with respect to the three separate muon systems, whilst reducing trigger rates and the occurrence of fake muons. The Global Muon Trigger also correlates muon candidates with regions in the calorimeter in order to confirm minimal ionization and to apply isolation criteria.

The CMS Global Trigger [11] at the top of the L1 Trigger chain issues the Level-1 accept/reject decision. It receives input from both the calorimeters and the muon system. The best four objects of each of the following categories, sorted by transverse energy or momentum and quality, are delivered by the Global Calorimeter and the Global Muon Trigger: isolated and non-isolated electrons or photons in a pseudorapidity range of $|\eta|<2.5$, central jets in $|\eta| \leq 3$, forward jets in $3<|\eta|<5, \tau$-jets in the central region up to $|\eta|<2.5$ and muons within $|\eta| \leq 2.1$ at the startup of LHC (later the coverage is foreseen to be enlarged to $|\eta| \leq 2.4$ ). The remaining trigger objects are the total $\left(\Sigma E_{T}\right)$ and missing transverse energies $\left(E_{T}\right)$, the transverse energy sum of all jets above a programmable threshold $\left(H_{T}\right)$ and the twelve jet multiplicities. A maximum number of 128 physics trigger algorithms running in parallel is possible. An algorithm is a logical combination of objects fulfilling energy, momentum or multiplicity threshold conditions. Topological requirements and veto conditions can also be applied. The algorithm settings are largely programmable.

\section{Physics Channels and Event Generation}

In agreement with the primary physics goals of CMS this study is concentrated on some of the main discovery channels. Supersymmetric processes that require in general multi-object triggers as opposed to simple conditions like single leptons or jets have been chosen. Specifically, sleptons $\left(\tilde{\ell} \tilde{\ell} \rightarrow \ell^{+} \ell^{-} \tilde{\chi}_{1}^{0} \tilde{\chi}_{1}^{0}\right)$, charginos/neutralinos $\left(\tilde{\chi}_{1}^{ \pm} \tilde{\chi}_{2}^{0} \rightarrow 3 \ell \tilde{\chi}_{1}^{0} \tilde{\chi}_{1}^{0}\right)$ and gluinos/squarks $(\tilde{g} / \tilde{q})$ belonging to this category were studied. The Drell-Yan produced sleptons and charginos/neutralinos lead to final states with multi-leptons and missing transverse energy, but no jets. For gluino/squark events in addition one or more jets can be required by the trigger. Although it is not a SUSY signal in the strict sense, WW-production has also been included. It is a benchmark process for triggering, since many particles predicted by existing theoretical models decay into intermediate vector bosons.

At the Snowmass 2001 "Summer Study on the Future of Particle Physics" a consensus was reached to define a list of SUSY models as benchmarks to be investigated in future collider studies [12,13]. Various scenarios, socalled "Snowmass Points and Slopes" (SPS), were proposed in terms of a few parameters describing "typical" to "extreme" R-parity conserving supersymmetry breaking mechanisms of mSUGRA (minimal supergravity model), GMSB (gauge mediated SUSY breaking model) and AMSB (anomaly mediated SUSY breaking model). All benchmark points respect current experimental constraints. By the time LHC starts in 2007, a significant integrated luminosity $\left(20 \mathrm{fb}^{-1}\right)$ is supposed to be collected at Fermilab's Tevatron collider. Its maximal sparticle mass reach for Run II [14] is assumed to be the starting point for LHC.

Channels with parameters according to selected Snowmass Points and Slopes and, in order to be compatible with HLT studies that focus on probing points at the upper mass reach of Tevatron Run II, general mSUGRA signals [15] provided by the CMS Physics Reconstruction and Selection Group were chosen. The parameters necessary to describe the selected points are $\tan \beta, m_{0}, m_{1 / 2}, \operatorname{sign}(\mu)$ and $A_{0} . \tan \beta$ is the ratio of the vacuum expectation values of the Higgs fields coupling to up- and down-like quarks. $m_{0}$ and $m_{1 / 2}$ are the universal scalar mass and universal gaugino mass at the GUT scale of approximately $2 \times 10^{16} \mathrm{GeV}$. $\operatorname{Sign}(\mu)$ is the sign of the Higgsino mass mixing parameter, and $A_{0}$ is the universal trilinear coupling. stop-quarks. The list of chosen signals is given in the following.

- Channel A (smuons at $S P S 1 a$ ):

$$
m_{0}=100 \mathrm{GeV}, m_{1 / 2}=250 \mathrm{GeV}, \operatorname{sign}(\mu)=+, \tan \beta=10, A_{0}=-100 \mathrm{GeV} \text {. }
$$


Subprocess: $\tilde{\mu}_{L} \tilde{\mu}_{L}$ with $\tilde{\mu}_{L}^{ \pm} \rightarrow \mu^{ \pm} \tilde{\chi}_{1}^{0}$

Sparticle mass spectrum:

$m_{\tilde{e}_{L}, \tilde{\mu}_{L}}=211 \mathrm{GeV}, m_{\tilde{\nu}_{L}}=196 \mathrm{GeV}, m_{\tilde{e}_{R}, \tilde{\mu}_{R}}=146 \mathrm{GeV}, m_{\tilde{\tau}_{1}}=146 \mathrm{GeV}$,

$m_{\tilde{g}}=562 \mathrm{GeV}, m_{\tilde{t}_{1}}=289 \mathrm{GeV}, m_{\tilde{u}_{L}}=501 \mathrm{GeV}, m_{\tilde{b}_{1}}=438 \mathrm{GeV}$,

$m_{\tilde{\chi}_{1}^{0}}=100 \mathrm{GeV}, m_{\tilde{\chi}_{2}^{0}}=189 \mathrm{GeV}, m_{\tilde{\chi}_{1}^{ \pm}}=188 \mathrm{GeV}, m_{\tilde{\chi}_{2}^{ \pm}}=398 \mathrm{GeV}$.

- Channel B (charginos/neutralinos at SPS 1a):

$m_{0}=100 \mathrm{GeV}, m_{1 / 2}=250 \mathrm{GeV}, \operatorname{sign}(\mu)=+, \tan \beta=10, A_{0}=-100 \mathrm{GeV}$.

Subprocess: $\tilde{\chi}_{2}^{0} \tilde{\chi}_{1}^{ \pm} \rightarrow\left(\tilde{\tau}_{1}^{ \pm} \tau^{\mp}\right)\left(\tilde{\tau}_{1}^{ \pm} \nu_{\tau}\right) \rightarrow\left(\tau^{+} \tau^{-} \tilde{\chi}_{1}^{0}\right)\left(\tau^{ \pm} \tilde{\chi}_{1}^{0} \nu_{\tau}\right) \rightarrow 3 l 2 \tilde{\chi}_{1}^{0} 4 \nu_{\tau} 3 \nu_{l}$ where $l=e$ or $\mu$.

Sparticle mass spectrum: as for channel A.

- Channel C (gluinos/squarks at $S P S 1 a$ ):

$m_{0}=100 \mathrm{GeV}, m_{1 / 2}=250 \mathrm{GeV}, \operatorname{sign}(\mu)=+, \tan \beta=10, A_{0}=-100 \mathrm{GeV}$.

Production processes: $\tilde{g} \tilde{g}, \tilde{g} \tilde{q}, \tilde{q} \tilde{q}$

Sparticle mass spectrum: as for channel A.

- Channel D (gluinos/squarks at $S P S$ 2):

$m_{0}=1450 \mathrm{GeV}, m_{1 / 2}=300 \mathrm{GeV}, \operatorname{sign}(\mu)=+, \tan \beta=10, A_{0}=0 \mathrm{GeV}$.

Production processes: $\tilde{g} \tilde{g}, \tilde{g} \tilde{q}, \tilde{q} \tilde{q}$

Sparticle mass spectrum:

$m_{\tilde{\ell}_{L}}=1456 \mathrm{GeV}, m_{\tilde{\ell}_{R}}=1452 \mathrm{GeV}, m_{\tilde{\tau}_{1}}=1439 \mathrm{GeV}$,

$m_{\tilde{g}}=784 \mathrm{GeV}, m_{\tilde{t}_{1}}=1003 \mathrm{GeV}, m_{\tilde{u}_{L}}=1532 \mathrm{GeV}, m_{\tilde{b}_{1}}=1297 \mathrm{GeV}$,

$m_{\tilde{\chi}_{1}^{0}}=122 \mathrm{GeV}, m_{\tilde{\chi}_{2}^{0}}=239 \mathrm{GeV}, m_{\tilde{\chi}_{1}^{ \pm}}=239 \mathrm{GeV}, m_{\tilde{\chi}_{2}^{ \pm}}=677 \mathrm{GeV}$.

- Channel E (mSUGRA4):

$m_{0}=20 \mathrm{GeV}, m_{1 / 2}=190 \mathrm{GeV}, \operatorname{sign}(\mu)=+, \tan \beta=10, A_{0}=0 \mathrm{GeV}$.

Sparticle mass spectrum:

$m_{h}=110 \mathrm{GeV}, m_{\tilde{g}}=466 \mathrm{GeV}, m_{\tilde{u}_{L}}=410 \mathrm{GeV}$,

$m_{\tilde{\chi}_{1}^{0}}=70 \mathrm{GeV}, m_{\tilde{\chi}_{2}^{0}}=126 \mathrm{GeV}, m_{\tilde{\chi}_{1}^{ \pm}}=125 \mathrm{GeV}, m_{\tilde{\chi}_{2}^{ \pm}}=296 \mathrm{GeV}$.

- Channel F (mSUGRA5):

$m_{0}=150 \mathrm{GeV}, m_{1 / 2}=180 \mathrm{GeV}, \operatorname{sign}(\mu)=+, \tan \beta=10, A_{0}=0 \mathrm{GeV}$.

Sparticle mass spectrum:

$m_{h}=110 \mathrm{GeV}, m_{\tilde{g}}=447 \mathrm{GeV}, m_{\tilde{u}_{L}}=415 \mathrm{GeV}$,

$m_{\tilde{\chi}_{1}^{0}}=66 \mathrm{GeV}, m_{\tilde{\chi}_{2}^{0}}=119 \mathrm{GeV}, m_{\tilde{\chi}_{1}^{ \pm}}=117 \mathrm{GeV}, m_{\tilde{\chi}_{2}^{ \pm}}=285 \mathrm{GeV}$.

- Channel G (mSUGRA6):

$m_{0}=300 \mathrm{GeV}, m_{1 / 2}=130 \mathrm{GeV}, \operatorname{sign}(\mu)=+, \tan \beta=10, A_{0}=0 \mathrm{GeV}$.

Sparticle mass spectrum:

$m_{h}=106 \mathrm{GeV}, m_{\tilde{g}}=349 \mathrm{GeV}, m_{\tilde{u}_{L}}=406 \mathrm{GeV}$,

$m_{\tilde{\chi}_{1}^{0}}=45 \mathrm{GeV}, m_{\tilde{\chi}_{2}^{0}}=80 \mathrm{GeV}, m_{\tilde{\chi}_{1}^{ \pm}}=77 \mathrm{GeV}, m_{\tilde{\chi}_{2}^{ \pm}}=233 \mathrm{GeV}$.

- Channel H (WW):

Subprocess: $\mathrm{W}^{+} \mathrm{W}^{-}$with $\mathrm{W} \rightarrow \mu \nu$ 
The position of the points chosen on the $m_{0}-m_{1 / 2}$ plane is shown in Fig. 1. The gluino/squark production channels are expected to show substantial missing transverse energies. In the case of $S P S 1 a$ (channels A, B and C) mostly leptons are expected, whilst for SPS 2 (channel D) jets will dominate. The mSUGRA4 channel (E) will be $\tau$-enriched and have a number of leptons. The characteristics of the mSUGRA5 channel (F) will be jets and leptons. The mSUGRA6 channel $(\mathrm{G})$ will have even more jets, but less missing transverse energy due to balanced contributions from several neutralinos and neutrinos. This fact is illustrated in Fig. 2, which shows the number of jets (central, forward and $\tau$-jets) and the missing transverse energy distributions of the mSUGRA samples as measured by the Level-1 Trigger.

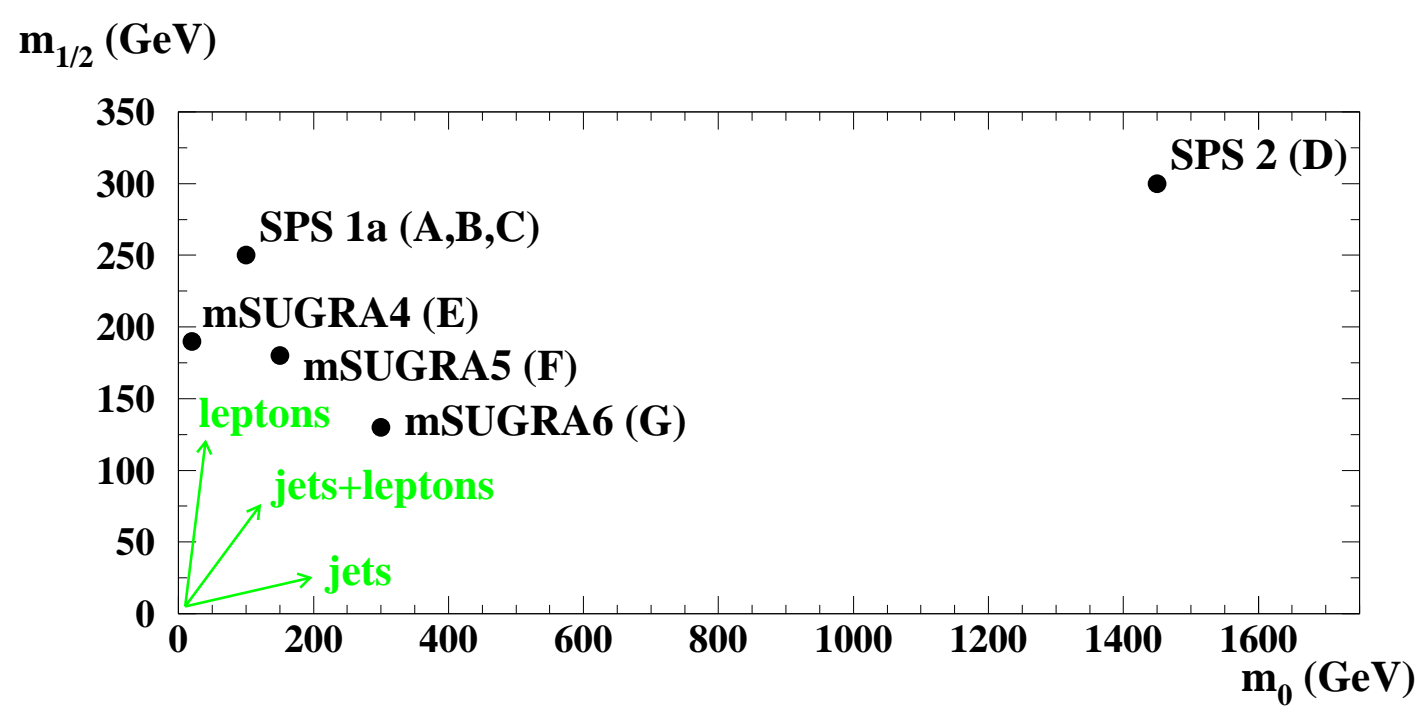

Figure 1: The position of the generated samples on the $m_{0}-m_{1 / 2}$ plane.

All physics channels were simulated with Monte Carlo event generator programs. PYTHIA $6.158[16,17]$ was used to simulate channels A, B, C, D and H. Channels E, F and G were generated with ISAJET 7.51 [18]. The proton parton distribution functions CTEQ 5L [19] were selected for both Monte Carlo programs. For each SUSY sample (channels A to G) 1000 events were generated, whilst for the WW sample (channel H) 2000 events were produced. The cross sections and the expected number of events of channels A to G after one LHC year corresponding to an integrated luminosity of $20 \mathrm{fb}^{-1}$ are summarized in Table 1 . The cross sections have been obtained with PYTHIA 6.158 (channels A to D) and ISAJET 7.51 (channels E to G).

Table 1: Cross sections and expected number of events of channels A to $\mathrm{G}$ for one year of LHC running $\left(20 \mathrm{fb}^{-1}\right)$.

\begin{tabular}{||c|l||c|c||}
\hline \hline Channel & Sample & Cross section $[\mathrm{pb}]$ & Number of events per LHC year \\
\hline \hline A & Smuons at $S P S 1 a$ & 0.110 & $2.20 * 10^{3}$ \\
\hline $\mathbf{B}$ & Charginos/neutralinos at $S P S 1 a$ & 0.267 & $5.34 * 10^{3}$ \\
\hline $\mathbf{C}$ & Gluinos/squarks at $S P S 1 a$ & 2360 & $4.72 * 10^{7}$ \\
\hline $\mathbf{D}$ & Gluinos/squarks at $S P S 2$ & 46.03 & $9.21 * 10^{5}$ \\
\hline $\mathbf{E}$ & mSUGRA4 & 191 & $3.82 * 10^{6}$ \\
\hline $\mathbf{F}$ & mSUGRA5 & 203 & $4.06 * 10^{6}$ \\
\hline $\mathbf{G}$ & mSUGRA6 & 600 & $1.20 * 10^{7}$ \\
\hline \hline
\end{tabular}

To reproduce the real conditions of data taking, with on average 3.5 proton-proton interactions (in-time pile-up) taking place per bunch crossing in a $25 \mathrm{~ns}$ time window, minimum bias events had to be added to each signal event. 

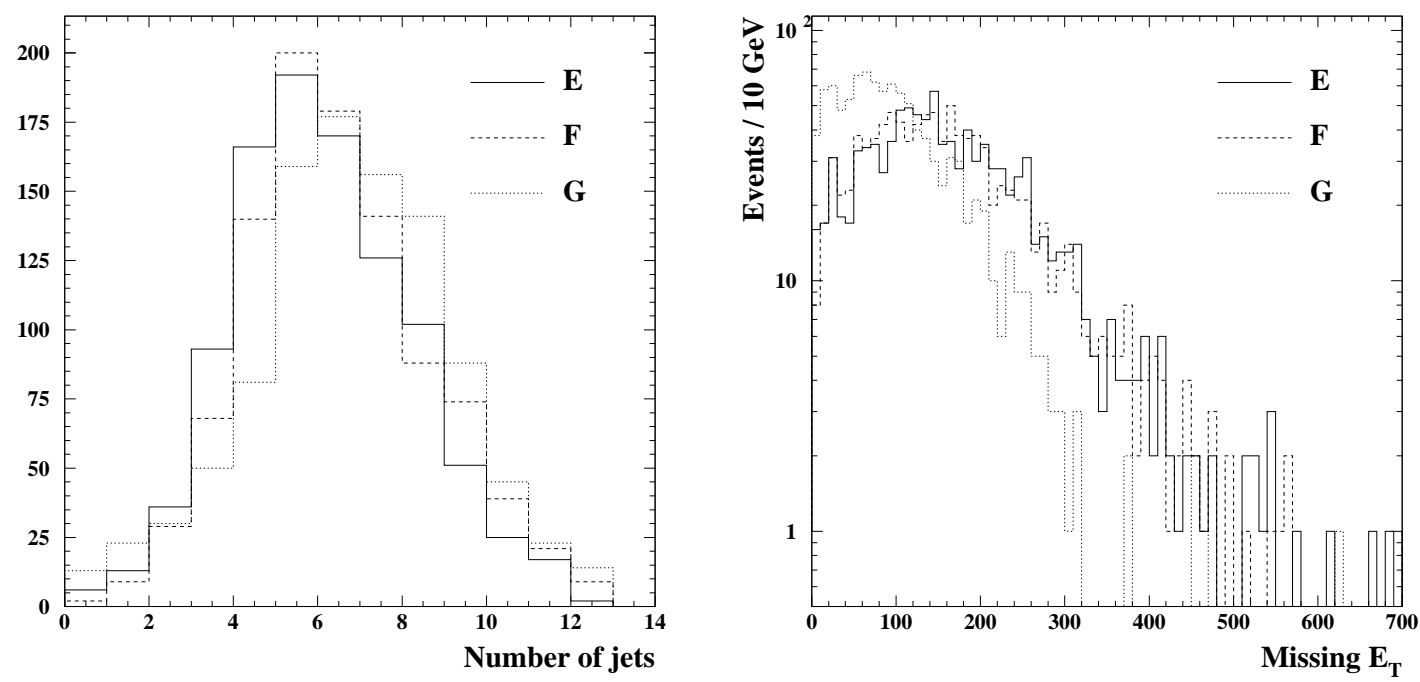

Figure 2: The number of jets (left) and the missing transverse energy distributions (right) of the mSUGRA channels E, F and G.

They were generated with PYTHIA 6.158.

\section{Detector and Trigger Simulation}

The simulation package CMSIM [20] (version 125), which describes the detector geometry and materials, was used to simulate hits in the CMS detector. A hit is described by the location, the magnitude and the time of occurrence of a particle's energy deposit in a detector. CMSIM simulates the effects of energy loss, multiple scattering and showering in the detector materials using the GEANT3 detector simulation tool [21]. The simulation was performed separately for the signal and the pile-up minimum bias samples.

The next step was to simulate the response of the detector readout electronics to the deposited energy, mixing signal and pile-up and adding electronics noise. The digitized detector hits corresponding to the raw data of a bunch crossing were produced within the Object-oriented Reconstruction for CMS Analysis (ORCA) framework (version 6), written in the $\mathrm{C}++$ programming language [22].

For the electromagnetic calorimeter, the duration of the reconstructed pulse is more than $200 \mathrm{~ns}$, thus several neighbouring bunch crossings overlap (out-of-time pile-up). Therefore the signals of five crossings before and three crossings after the triggered bunch crossing were superposed. The time of an interaction is by definition at the maximum of the signal.

The input for the L1 jet trigger are the calorimeter digitizations. The brass/scintillator hadronic calorimeter [23] has a non-linear response, i.e. the average reconstructed energy is inferior to the actual energy deposit due to the non-compensating nature of the brass/scintillator samples. The non-linearity is larger if the energy deposit is smaller. Hadronic showers were simulated using the GCALOR hadronic shower generator [24] with energy cutoffs for photons, electrons, neutral and charged hadrons tuned according to test beam measurements [25]. The non-linearity is well reproduced in the simulation.

As foreseen in the L1 Calorimeter Trigger hardware implementation, the non-linearity of the hadronic energy measurement was corrected by correction functions depending on transverse energy and pseudorapidity. The pileup adds energy to the signal, thus decreasing the non-linearity. This effect is bigger at high luminosity than at low luminosity, therefore the correction function also depends on the luminosity. The high resolution crystal electromagnetic calorimeter [26] has a quite linear response. 
Muons are reconstructed separately in the barrel drift tube or endcap cathode strip precision chambers and in the RPC trigger chambers. The response of the time-to-digital converters (TDC) form the output of the digitization step of the barrel drift tube system. Muon direction and impact position with respect to the sense wire and effects of the residual magnetic field in the air gaps of the magnet iron yoke, where the chambers are located, are taken into account in the simulation [27]. The TDC output signal for the hit reconstruction is obtained from the drift time by adding the muon time-of-flight from the collision vertex and the propagation time of the signal along the cell wire. In case of multiple hits in a cell due to delta rays and/or muon showering, the signal from the hit nearest the wire is retained.

The digitization step of the cathode strip chamber system involves simulating the response of the analog-to-digital converters and discriminators connected to the strips and wires respectively. A detailed description of the simulation, which includes cross-talk and electronic noise, is given in ref. [28].

The RPC response is assumed to take place within $20 \mathrm{~ns}$ of the passage of a charged particle through the detector with a 3 ns Gaussian distributed jitter, which also accounts for the contribution of the front-end electronics and the signal transfer along the cables. The $20 \mathrm{~ns}$ wide time gates were adjusted in order to accommodate triggering signals. The RPC cluster size is set to 1.5 strips, and the average hit efficiency is $95 \%$ [29]. It should be noted that intrinsic chamber noise, to which RPC chambers are most susceptible, is not included in the simulation because of CPU time constraints.

The performance of the CMS Level-1 Trigger system has been simulated using the ORCA framework. It contains a detailed description of the L1 Trigger hardware, mapped into the object-oriented software structure in such a way that each component is represented by a $\mathrm{C}++$ object. Emphasis was put on designing a simulation which closely follows the hardware structure. The simulation models the hierarchy of components from FPGA (field programmable gate arrays) or ASIC (application specific integrated circuits) chip level, through electronics boards, up to the L1 Trigger subsystems. A description of the various hardware components was implemented in C++ to exactly mimic the functionality of currently constructed prototypes as described in [1]. Bit-wise arithmetic has been used whenever feasible in the simulation in order to reproduce the real electronic circuit behaviour as accurately as possible [30].

\section{Trigger Scenario}

In accordance with the capacity of the HLT event filter and the data acquisition (DAQ) at the startup of LHC during the initial low luminosity running, the maximum output rate of the Level-1 Trigger has been set to $50 \mathrm{kHz}$. The cross section of the background has been estimated by Monte Carlo studies, and it is assumed that the uncertainty of the result is about a factor of two. CMS has decided to use a safety factor of three, allowing a total Level-1 output rate of approximately $16 \mathrm{kHz}$ dedicated to physics triggers.

A basic trigger menu with the trigger conditions shown in Table 2 has been defined. The thresholds for the trigger objects electrons, muons, jets, $\tau$-jets, missing and total transverse energies are chosen to yield the permitted total rate of approximately $16 \mathrm{kHz}$. The muon thresholds originate from ref. [2]. It is emphasized that the described trigger menu has not been optimized for Supersymmetry in general nor for any specific supersymmetric channel. It represents a multi-purpose menu suitable for any new particle searches, except for heavy ion or B-physics studies, which will require for example a lowering of the muon thresholds.

The bandwidth quota of $16 \mathrm{kHz}$ has been divided into four equal groups among the $e / e e$, the $\mu / \mu \mu$, the $\tau / \tau \tau$ triggers and the rest, which includes jet triggers, $E_{T}$-sums and combined triggers. Most of the quota has been assigned to single and double object triggers, since they yield the largest contribution to the signal efficiencies. Multiple (more than two objects) and combined (more than one type of objects) triggers have lower backgrounds and modest contributions to the signal efficiencies. About $4 \mathrm{kHz}$ are allocated to each of the four trigger groups $e / e e, \mu / \mu \mu, \tau / \tau \tau$ and the jet and combination triggers. The trigger rates obtained are $3.6 \mathrm{kHz}$ for the muon group, $4.3 \mathrm{kHz}$ for the electron group, $3.0 \mathrm{kHz}$ for the $\tau$-group and $3.0 \mathrm{kHz}$ for the pure jet triggers of the fourth group made of jet and combination triggers. The overall total trigger rate is smaller than the sum of the rates of the four groups since overlaps are possible. The thresholds are a working hypothesis for the nominal maximum initial LHC 
luminosity of $2 \times 10^{33} \mathrm{~cm}^{-2} \mathrm{~s}^{-1}$. They will have to be adapted to the actual luminosity conditions during the course of each data taking period. At lower instantaneous luminosities the thresholds could be lowered as well, which leads to higher Level-1 trigger efficiencies but generally also to more background in the data sample selected by the Level-1 Trigger. The thresholds for the first three groups and the pure jet trigger thresholds of the fourth group for the nominal low LHC luminosity are not expected to change significantly in the future. The thresholds for the combined triggers may undergo more retuning, which would affect the individual rate distributions in that group. However, the additional contribution to the overall rate is small compared to that of the single and double object triggers.

Table 2: Trigger conditions and thresholds.

\begin{tabular}{||l|c||}
\hline \hline Trigger conditions & Thresholds [GeV] \\
\hline \hline$\mu$ & 14 \\
\hline$\mu \mu$ & 3 \\
\hline \hline$e e$ isolated & 23 \\
\hline$e e$ all & 12 \\
\hline \hline$\tau$ & 19 \\
\hline$\tau \tau$ & 93 \\
\hline \hline$J$ & 66 \\
\hline $3 J$ & 138 \\
\hline $4 J$ & 66 \\
\hline$J E_{T}$ & 53 \\
\hline$\mu e$ & 60,65 \\
\hline$\mu \tau$ & 4,13 \\
\hline$\mu J$ & 4,55 \\
\hline$\mu E_{T}$ & 4,50 \\
\hline$\mu \Sigma E_{T}$ & 4,45 \\
\hline$e J$ & 4,300 \\
\hline$e \tau$ & 15,100 \\
\hline$E_{T}$ & 15,70 \\
\hline$e E_{T}$ & 140 \\
\hline$\Sigma E_{T}$ & 20,75 \\
\hline$H_{T}$ & 600 \\
\hline \hline
\end{tabular}

\section{Results}

The goal of this study was to determine Level-1 trigger efficiencies for selected SUSY events recorded during the initial low luminosity running period of the LHC. Good L1 efficiencies are a prerequisite to good overall efficiencies, which are a convolution of both Level-1 and HLT efficiencies.

The Level-1 trigger efficiency is defined as the ratio of the number of events accepted by the Global Trigger in the fiducial volume equipped with trigger electronics and the number of generated events. No geometrical restrictions were applied to the particles in the generation process. Once real data are available, they will be used to determine trigger efficiencies independently from simulated events. The principle will be to operate with overlapping triggers running in parallel. These can be obtained in several ways. One possibility would be to reduce energy or momentum thresholds, another to relax constraints such as electron or muon isolation requirements. If the trigger rates become too high with the loosened criteria, prescaling of a trigger is possible.

The Level-1 results obtained with the generated Monte Carlo samples are summarized in Tables 3 and 4 . The individual, the additional and the exclusive efficiencies of every trigger have been calculated for each channel. The 
Table 3: Efficiencies at the Global Trigger for mSUGRA channels at the upper mass reach of Tevatron II.

\begin{tabular}{|c|c|c|c|c|c|c|c|c|c|c|}
\hline Trigger & Thresholds & \multicolumn{3}{|c|}{$\begin{array}{l}\varepsilon_{E ; m_{0}=20}^{m_{1 / 2}=190}[\%] \\
\end{array}$} & \multicolumn{3}{|c|}{$\begin{array}{c}\varepsilon_{1 / 2}=180 \\
\varepsilon_{F ; m_{0}}=150[\%] \\
\end{array}$} & \multicolumn{3}{|c|}{$\begin{array}{c}\varepsilon_{1 / 2}^{m_{1 / 2}}=130 \\
\varepsilon_{G ; m_{0}}=300[\%] \\
\end{array}$} \\
\hline & {$[\mathrm{GeV}]$} & ind. & add. & exc. & ind. & add. & exc. & ind. & add. & exc. \\
\hline$\mu$ & 14 & 18.5 & 18.5 & 0.5 & 18.9 & 18.9 & 0.1 & $\overline{17.2}$ & $\overline{17.2}$ & 0.5 \\
\hline$\mu \mu$ & 3 & 7.4 & 1.6 & 0 & 6.9 & 1.1 & 0 & 5.0 & 0.9 & 0 \\
\hline$e$ & 23 & 12.0 & 10.2 & 0.3 & 12.5 & 10.3 & 0.1 & 10.6 & 9.2 & 0.2 \\
\hline$e e$ isol. & 12 & 2.9 & 1.1 & 0.1 & 4.5 & 0.9 & 0 & 4.6 & 1.7 & 0 \\
\hline$e e$ all & 19 & 4.5 & 3.3 & 0 & 4.4 & 3.0 & 0 & 5.9 & 4.9 & 0.2 \\
\hline$J$ & 138 & 82.8 & 53.8 & 0.6 & 86.8 & 56.8 & 0.1 & 70.1 & 44.7 & 0.1 \\
\hline $3 J$ & 66 & 55.9 & 2.5 & 0.3 & 61.5 & 1.7 & 0.1 & 63.8 & 6.1 & 0.3 \\
\hline $4 J$ & 53 & 38.3 & 0.3 & 0 & 46.1 & 0.4 & 0 & 54.9 & 1.3 & 0.2 \\
\hline$J E_{T}$ & 60,65 & 85.3 & 3.5 & 1.7 & 84.2 & 2.6 & 0.7 & 64.6 & 1.9 & 0.8 \\
\hline$\tau$ & 93 & 46.6 & 0.6 & 0.4 & 49.8 & 0.4 & 0.3 & 46.2 & 0.5 & 0.4 \\
\hline$\tau \tau$ & 66 & 26.7 & 0.2 & 0.1 & 29.0 & 0 & 0 & 34.0 & 0.2 & 0.1 \\
\hline$\mu e$ & 4,13 & 6.2 & 0.1 & 0.1 & 6.6 & 0 & 0 & 7.6 & 0.1 & 0 \\
\hline$\mu \tau$ & 4.55 & 21.7 & 0.2 & 0 & 22.0 & 0 & 0 & 24.3 & 0.1 & 0 \\
\hline$\mu J$ & 4,50 & 29.5 & 0.3 & 0 & 29.1 & 0.2 & 0.1 & 29.1 & 0.1 & 0.1 \\
\hline$\mu E_{T}$ & 4,45 & 27.5 & 0 & 0 & 27.1 & 0 & 0 & 24.3 & 0 & 0 \\
\hline$\mu \sum E_{T}$ & 4,300 & 21.6 & 0 & 0 & 25.0 & 0 & 0 & 22.7 & 0 & 0 \\
\hline$e J$ & 15,100 & 18.4 & 0 & 0 & 18.0 & 0 & 0 & 17.7 & 0 & 0 \\
\hline$e \tau$ & 15,70 & 14.5 & 0 & 0 & 14.7 & 0 & 0 & 14.9 & 0 & 0 \\
\hline$E_{T}$ & 140 & 54.9 & 0 & 0 & 53.3 & 0 & 0 & 24.5 & 0 & 0 \\
\hline$e E_{T}$ & 20,75 & 12.4 & 0 & 0 & 10.6 & 0 & 0 & 7.9 & 0 & 0 \\
\hline$\Sigma E_{T}$ & 600 & 29.1 & 0 & 0 & 33.1 & 0 & 0 & 22.4 & 0 & 0 \\
\hline$H_{T}$ & 400 & 50.6 & 0 & 0 & 57.1 & 0 & 0 & 43.0 & 0 & 0 \\
\hline Total & ficiency & & 96.2 & & & 96.3 & & & 88.9 & \\
\hline
\end{tabular}

individual efficiency is the efficiency of a trigger as if it were the only trigger applied. In reality several triggers are running in parallel and overlap, i.e. the same event can be selected by more than one trigger. This overlap is excluded by the additional trigger efficiency, which does not count events already found by a previous trigger. The order of triggers as listed in Tables 3 and 4 was used. The exclusive efficiency refers to events found by one and only one trigger, i.e. events found by more than one trigger are not counted here, regardless whether the trigger is previous or subsequent.

The additional and exclusive efficiencies are very important indicators of the usefulness of the particular triggers. The sum of the additional triggers is the overall efficiency of the trigger system for a physics channel. Whilst a particular additional efficiency depends on the order of the triggers, the sum of them is independent from it.

The trigger efficiencies for mSUGRA points without specific subprocess constraints are good $-88 \%$ to $96 \%$ for the mSUGRA channels E, F and G (Table 3). Specific final states show a bigger spread in efficiencies (Table 4 for channels A to D and $\mathrm{H})-72 \%$ to $100 \%$.

Channel B has the lowest efficiency of all. It has three charged leptons (electrons or muons), two neutralinos and seven neutrinos in the final state, coming from the decay of the $\tilde{\chi}_{2}^{0} \tilde{\chi}_{1}^{ \pm}$pair. Despite the good electron and muon detection capacity of the CMS detector, it has only $72.3 \%$ efficiency, even though the leptons are produced from two initial charginos and neutralinos of about $200 \mathrm{GeV}$ mass. In those events which are rejected by the trigger, either the electrons have very low $E_{T}$ (Figs. 3 and 4) or the pseudorapidities of the muons are beyond the $|\eta|<2.1$ coverage of the muon trigger (Figs. 5 and 6 ).

It was investigated whether a substantial fraction of the energy is carried by invisible particles and whether a trigger on missing transverse energy could be used in consequence. A dielectron plus missing $E_{T}$ trigger with symmetric 


\begin{tabular}{|c|c|c|c|c|c|c|c|c|c|c|c|c|c|c|c|c|}
\hline Trigger & Thresholds & \multicolumn{3}{|c|}{$\varepsilon_{A}\left(\tilde{\mu}_{L} \tilde{\mu}_{L}\right)[\%]$} & \multicolumn{3}{|c|}{$\varepsilon_{B}\left(\tilde{\chi}_{2}^{0} \tilde{\chi}_{1}^{ \pm}\right)[\%]$} & \multicolumn{3}{|c|}{$\begin{array}{l}\varepsilon_{C ; m_{0}=100}^{m_{1 / 2}=250}[\%] \\
\end{array}$} & \multicolumn{3}{|c|}{ 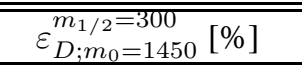 } & \multicolumn{3}{|c|}{$\begin{array}{l}\varepsilon_{H}(W W)[\%] \\
\end{array}$} \\
\hline & $\begin{array}{l}\mathrm{GeV}] \\
\end{array}$ & ind. & add. & $\begin{array}{l}\text { exc. } \\
\end{array}$ & בind. & add. & exc. & ind. & add. & 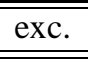 & ind. & add. & 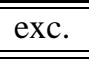 & "ind. & בadd. & exc. \\
\hline$\overline{\mu \mu}$ & $\overline{14}$ & 92.5 & 992.5 & 16.7 & 449.0 & 449.0 & 12.3 & 27.8 & 27.8 & 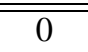 & $\overline{440.8}$ & 40.8 & 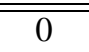 & 80.5 & 80.5 & 33.5 \\
\hline$\mu \mu$ & 3 & 68.8 & 0.1 & 0.0 & 24.6 & 2.9 & 1.8 & 13.8 & 1.3 & 0 & 24.3 & 3.6 & 0 & 43.4 & 0.1 & 0.1 \\
\hline$e$ & 23 & 3.3 & 0.3 & 0.3 & 22.9 & 13.1 & 4.2 & 19.7 & 14.9 & 0 & 20.2 & 11.7 & 0 & 0.9 & 0.2 & 0.1 \\
\hline$e e$ isol. & 12 & 0.5 & 0 & 0 & 8.9 & 1.9 & 1.2 & 8.6 & 0.5 & 0 & 7.1 & 0.6 & 0 & 0.1 & 0 & 0 \\
\hline$e e$ all & 19 & 0 & 0 & 0 & 0 & 0 & 0 & 5.9 & 3.5 & 0 & 10.0 & 5.3 & 0 & 0 & 0 & 0 \\
\hline $\bar{J}$ & 138 & 3.7 & 0.2 & $\overline{0}$ & 1.4 & 0.2 & 0 & \begin{tabular}{|l|}
88.4 \\
\end{tabular} & 447.0 & 0.1 & 98.7 & 37.7 & $\overline{0}$ & 0.2 & 0.1 & $\overline{0}$ \\
\hline $3 J$ & 66 & 0.1 & $\overline{0}$ & 0 & 0.2 & $\overline{0}$ & 0 & 68.6 & 1.5 & 0.1 & 97.6 & 0.3 & 0 & $\overline{0}$ & 0 & $\overline{0}$ \\
\hline $4 J$ & 53 & 0.1 & 0 & 0 & 0.1 & 0 & 0 & 53.1 & 0.3 & 0.2 & 94.8 & 0 & 0 & 0 & 0 & 0 \\
\hline$J E_{T}$ & 60,65 & 7.8 & 0.3 & 0.2 & 5.3 & 0.8 & 0.4 & 84.8 & 0.8 & 0.4 & 92.2 & 0 & 0 & 0.6 & 0.1 & 0.1 \\
\hline $\bar{\tau}$ & 93 & 4.9 & $\overline{0}$ & 0 & 4.4 & 0.2 & 0.1 & 55.2 & 0.1 & 0 & 68.2 & 0 & 0 & 0.5 & 0 & $\overline{0}$ \\
\hline$\tau \tau$ & 66 & 0.6 & 0 & 0 & 0.9 & 0 & 0 & 33.2 & 0.1 & 0 & 51.0 & 0 & 0 & 0.1 & 0 & 0 \\
\hline$\mu e$ & 4,13 & 5.0 & 0 & 0 & 26.0 & 2.7 & 2.3 & 11.1 & 0 & 0 & 19.3 & 0 & 0 & 2.2 & 0.1 & 0.1 \\
\hline$\mu \tau$ & 4,55 & 14.3 & 0 & 0 & 14.2 & 0.4 & 0 & 31.6 & 0 & 0 & 53.2 & 0 & 0 & 2.6 & 0.2 & 0 \\
\hline$\mu J$ & 4,50 & 25.1 & 0 & 0 & 22.7 & 0.9 & 0.7 & 38.6 & 0.1 & 0 & 59.1 & 0.1 & 0 & 6.5 & 0.1 & 0.1 \\
\hline$\mu E_{T}$ & 4,45 & 13.6 & 0 & 0 & 9.3 & 0.1 & 0.1 & 35.1 & 0 & 0 & 56.9 & 0 & 0 & 2.0 & 0.1 & 0.1 \\
\hline$\mu \Sigma E_{T}$ & 4,300 & 2.2 & 0 & 0 & 1.3 & 0 & 0 & 34.1 & 0 & 0 & 58.8 & 0 & 0 & 1.2 & 0 & 0 \\
\hline$e J$ & 15,100 & 1.2 & 0 & $\overline{0}$ & 3.7 & $\overline{0}$ & 0 & 26.5 & 0 & $\overline{0}$ & 28.4 & 0 & $\overline{0}$ & 0.1 & 0 & 0 \\
\hline$e \tau$ & 15,70 & 1.4 & $\overline{0}$ & 0 & 8.4 & 0.1 & 0.1 & 23.2 & 0 & 0 & 24.9 & 0 & 0 & 0.4 & 0 & 0 \\
\hline$E_{T}$ & 140 & 1.7 & 0 & 0 & 0.3 & $\overline{0}$ & 0 & 61.1 & 0 & 0 & 67.5 & 0 & 0 & 0.1 & 0 & 0 \\
\hline$e E_{T}$ & 20,75 & 1.2 & 0 & 0 & 2.1 & 0 & 0 & 18.5 & 0 & 0 & 20.1 & 0 & 0 & 0.2 & 0 & 0 \\
\hline$\Sigma E_{T}$ & 600 & 0.1 & 0 & 0 & 0 & 0 & 0 & 39.3 & 0 & 0 & 86.2 & 0 & 0 & 0.1 & 0 & 0 \\
\hline$H_{T}$ & 400 & 0.1 & 0 & 0 & 0 & 0 & 0 & 64.5 & 0 & 0 & 96.3 & 0 & 0 & 0 & 0 & 0 \\
\hline \multicolumn{2}{|c|}{ Total Efficiency } & & \begin{tabular}{|c|}
93.4 \\
\end{tabular} & & & 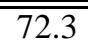 & & & 497.9 & & & $\begin{array}{l}100.0 \\
\end{array}$ & & & 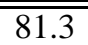 & \\
\hline
\end{tabular}



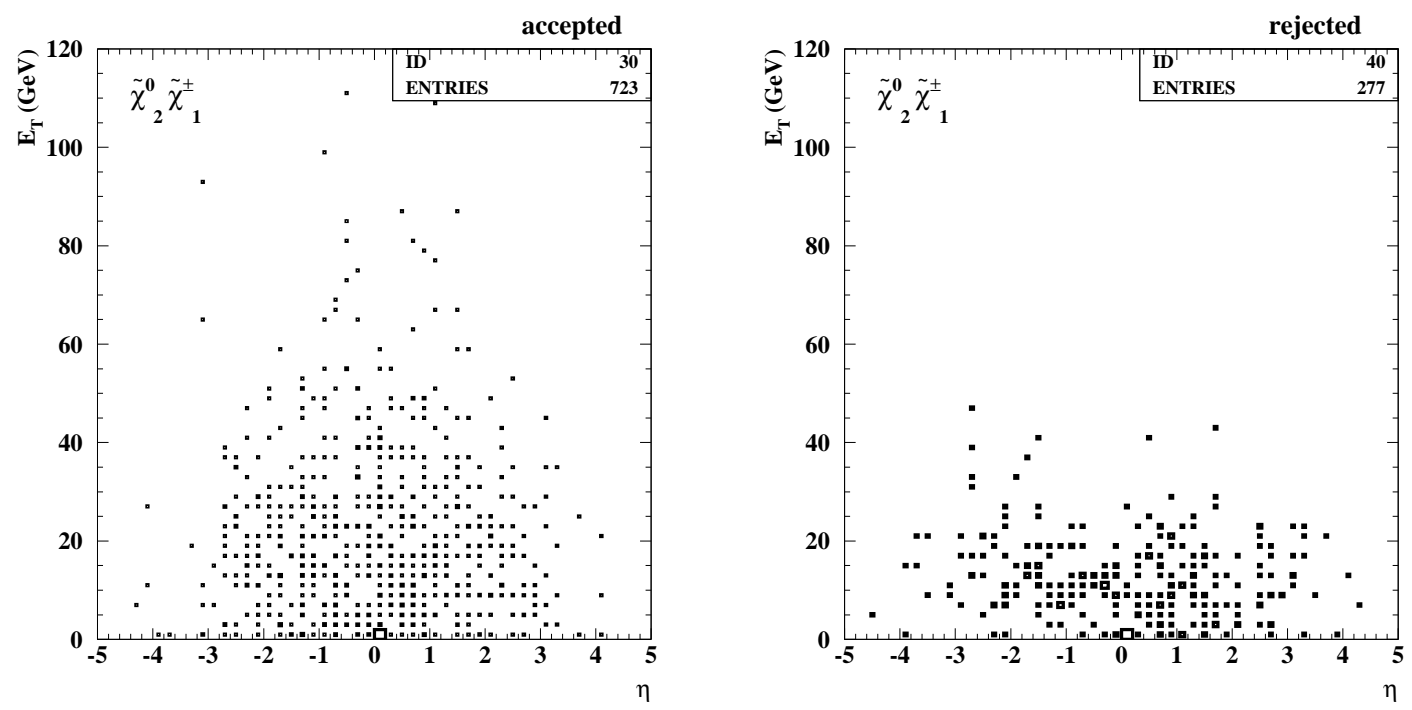

Figure 3: $E_{T}$ vs. $\eta$ distribution of the generated (PYTHIA) highest energy electrons in $\tilde{\chi}_{2}^{0} \tilde{\chi}_{1}^{ \pm}$events accepted (left) and rejected (right) by the L1 Trigger.
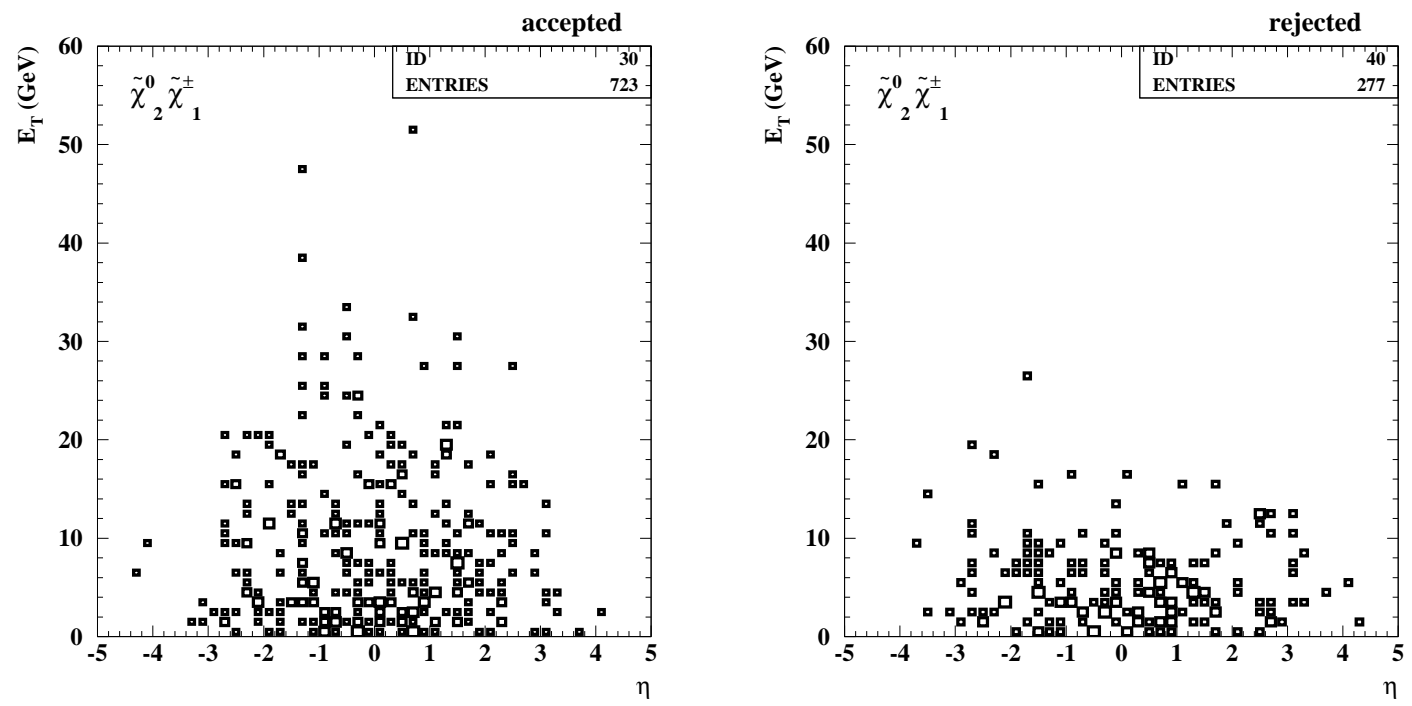

Figure 4: $E_{T}$ vs. $\eta$ distribution of the generated (PYTHIA) second highest energy electrons in $\tilde{\chi}_{2}^{0} \tilde{\chi}_{1}^{ \pm}$events accepted (left) and rejected (right) by the L1 Trigger. 

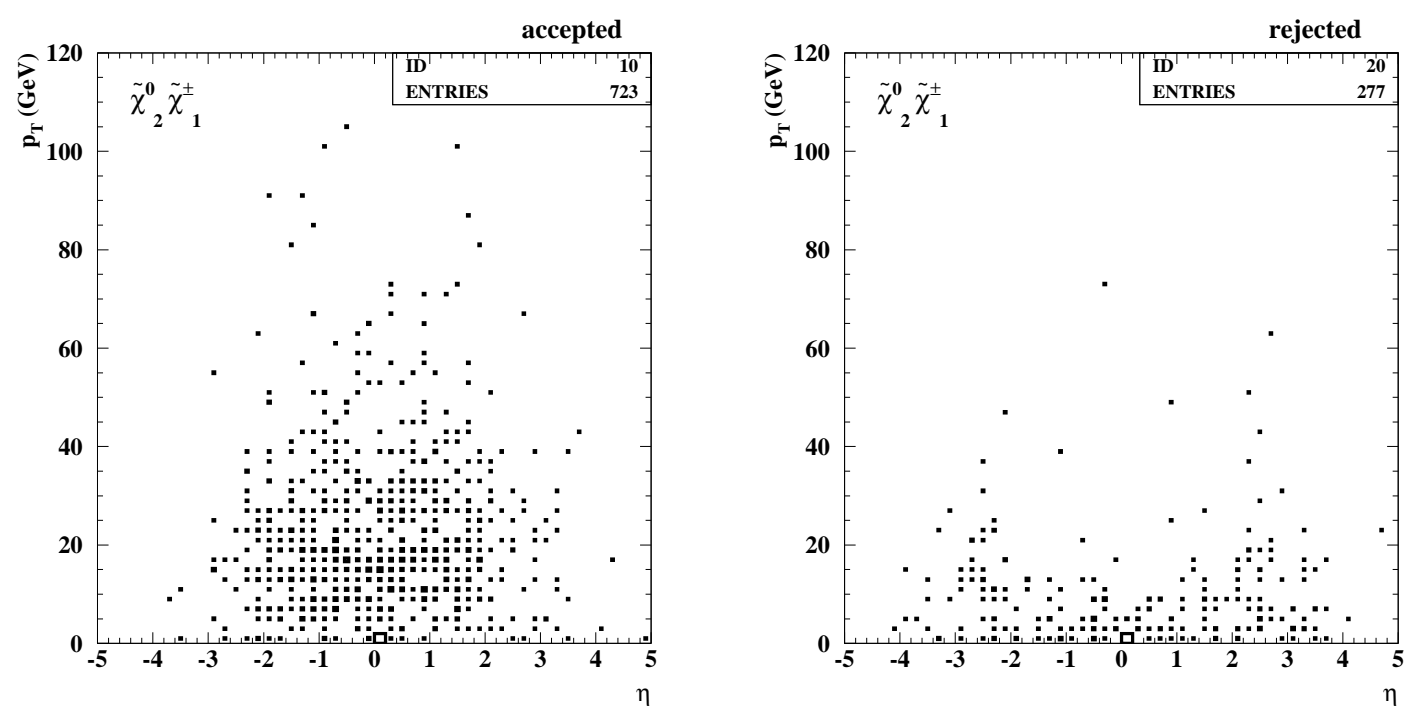

Figure 5: $p_{T}$ vs. $\eta$ distribution of the generated (PYTHIA) highest momentum muons in $\tilde{\chi}_{2}^{0} \tilde{\chi}_{1}^{ \pm}$events accepted (left) and rejected (right) by the L1 Trigger.
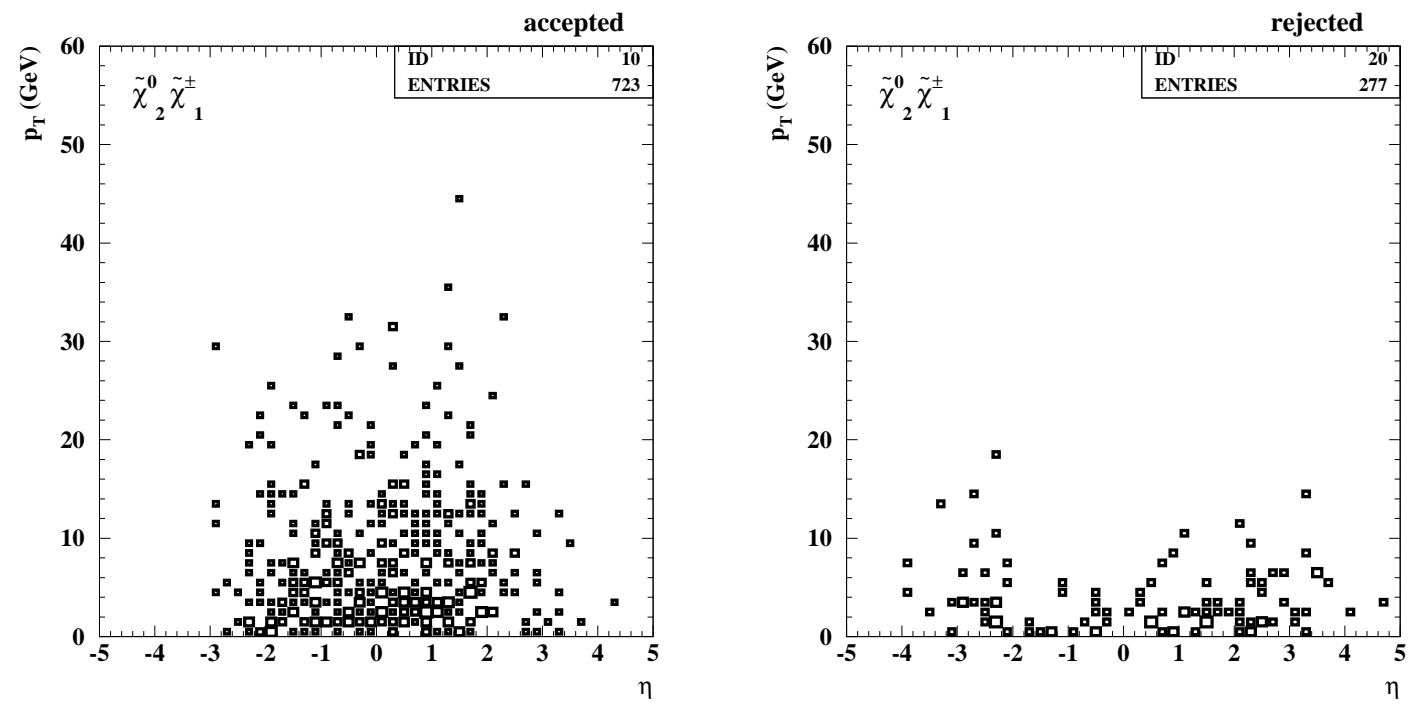

Figure 6: $p_{T}$ vs. $\eta$ distribution of the generated (PYTHIA) second highest momentum muons in $\tilde{\chi}_{2}^{0} \tilde{\chi}_{1}^{ \pm}$events accepted (left) and rejected (right) by the L1 Trigger. 
thresholds of $8 \mathrm{GeV}$ for the two electrons and $20 \mathrm{GeV}$ for $\mathbb{E}_{T}$ respectively was used for this investigation. The scalar energy sum and the vector transverse energy sum of the invisible particles mentioned above were checked, and it was found that the energy sum was bigger for the rejected events than for those passing the trigger, while the $E_{T}$-sum was approximately the same (Fig. 7). This means that the invisible particles in the rejected events tend to
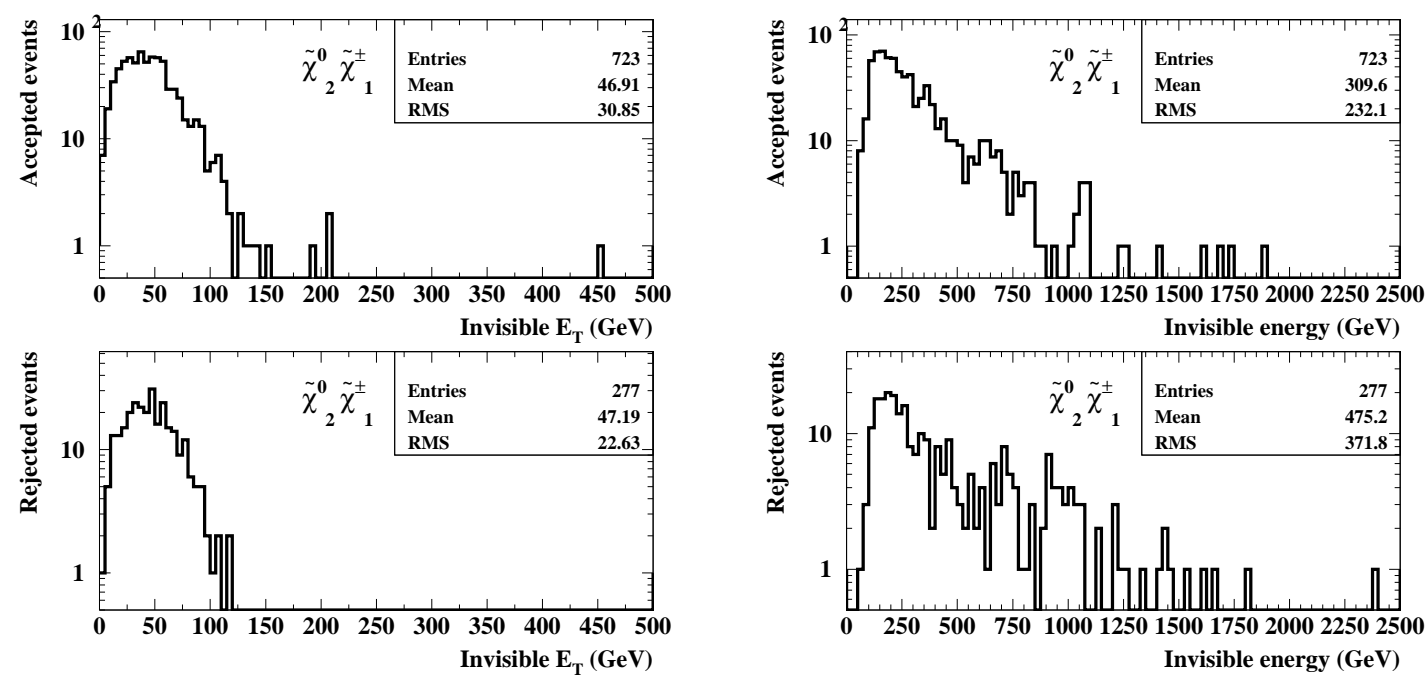

Figure 7: The generated $E_{T}$ sums (left) and energy sums (right) of invisible particles of the $\tilde{\chi}_{2}^{0} \tilde{\chi}_{1}^{ \pm}$events accepted (top) and rejected (bottom) by the L1 Trigger.

balance their contributions on the $E_{T}$-plane, although much energy is carried by them. There are many of those weakly interacting particles in the event, therefore the probability of such a cancellation effect is high.

The trigger efficiencies listed in Tables 3 and 4 are functions of the choice of thresholds, of the energy or momentum resolution and geometrical acceptance of the $\mathrm{L} 1$ Trigger. In order to give a qualitative appreciation of the different effects two channels, A and C, were specifically investigated with regard to the muon trigger. The smuon channel A has a high muon trigger efficiency, $92.5 \%$ for the single muon trigger and $68.8 \%$ for the dimuon trigger respectively. The corresponding numbers for the gluino/squark channel C decaying predominantly into leptons are only $27.8 \%$ and $13.8 \%$.
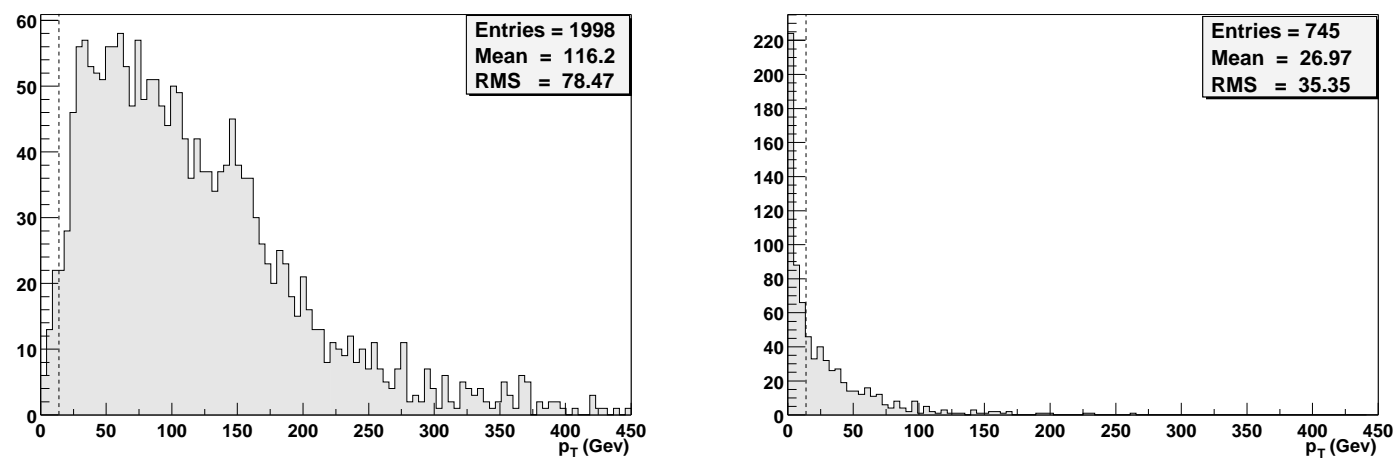

Figure 8: $p_{T}$-distributions of the two highest- $p_{T}$ final state muons for the smuon channel $\mathrm{A}(l e f t)$ and the gluino/squark channel C (right). 
Fig. 8 shows the transverse momentum spectra of the two highest- $p_{T}$ final state muons tracked through the CMS detector for the smuon channel A and for the gluino/squark channel $\mathrm{C}$. The corresponding transverse momenta of the two highest $-p_{T}$ muon candidates at the output of the L1 Global Muon Trigger are shown in Fig. 9. The
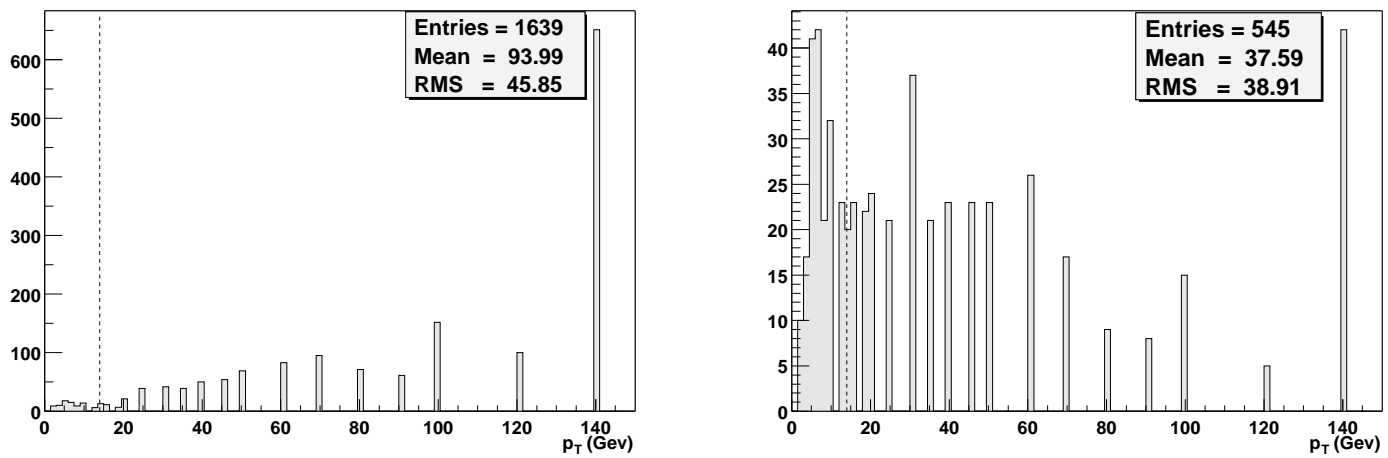

Figure 9: $p_{T}$-distributions of the two highest- $p_{T}$ L1 muon candidates for the smuon channel A (left) and the gluino/squark channel C (right).

dashed line indicates the $p_{T}$-threshold of $14 \mathrm{GeV}$ for the single muon trigger. In the L1 Muon Trigger a non-linear $p_{T}$-scale with $32 p_{T}$-intervals is used. The intervals are more closely spaced at lower than at higher $p_{T}$-values. All muon candidates with transverse momenta higher than $140 \mathrm{GeV}$ are assigned to the $140 \mathrm{GeV}$ bin.

The high single muon trigger efficiency for the smuon channel A is obviously due to the high momenta of the original muons. For the gluino/squark channel $\mathrm{C}$, which has fewer muons than channel A to start with, many muons do not pass the threshold of $14 \mathrm{GeV}$. For both channels losses due to the limited $\eta$-coverage of the muon trigger play a less important role, as can be seen from Figs. 10 and 11, which show the pseudorapidity distributions for channels $\mathrm{A}$ and $\mathrm{C}$ for the final state muons and for the L1 muon candidates respectively. The dashed lines delimit the $\eta$-coverage of the muon trigger.
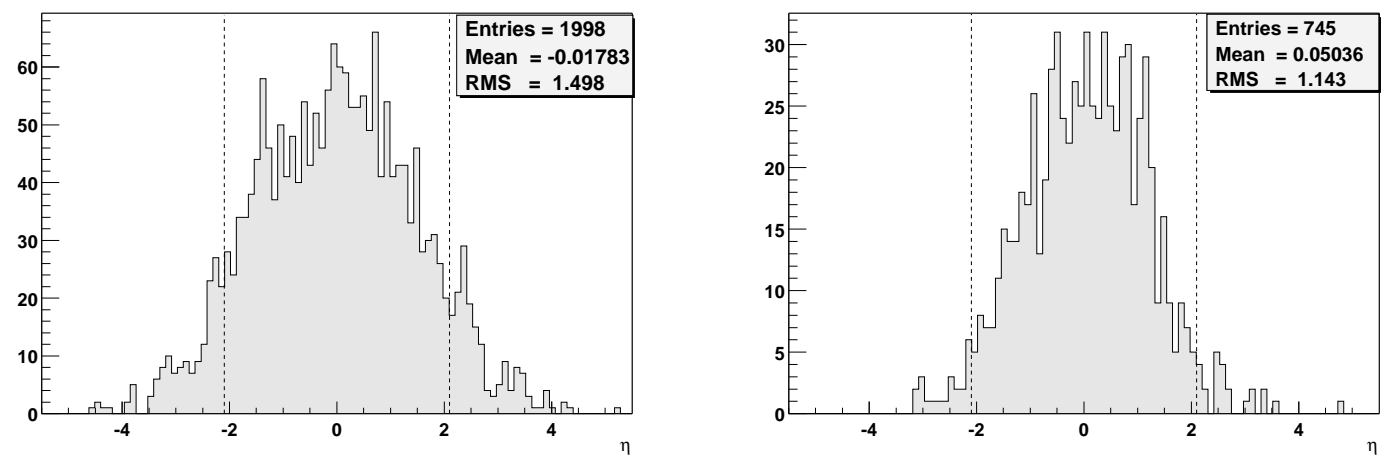

Figure 10: $\eta$-distributions of the two highest- $p_{T}$ final state muons for the smuon channel A (left) and the gluino/squark channel C (right).

In order to appreciate the real physics potential of CMS the convoluted Level-1 and High Level Trigger efficiencies have to be calculated. Combined L1 and HLT efficiencies for some supersymmetry scenarios have been estimated in ref. [2]. The actual values depend very much on the chosen trigger parameters. For example, mSUGRA4 triggered by the jet plus missing energy algorithm with $\mathrm{L} 1$ thresholds of $79 \mathrm{GeV}$ and $46 \mathrm{GeV}$ respectively, shows an L1 efficiency of 88\%. The combined L1/HLT efficiency for HLT cuts of $180 \mathrm{GeV}$ for the jet and $123 \mathrm{GeV}$ for the missing energy is $67 \%$. mSUGRA6 goes from an L1 efficiency of $71 \%$ to a combined L1/HLT efficiency of 

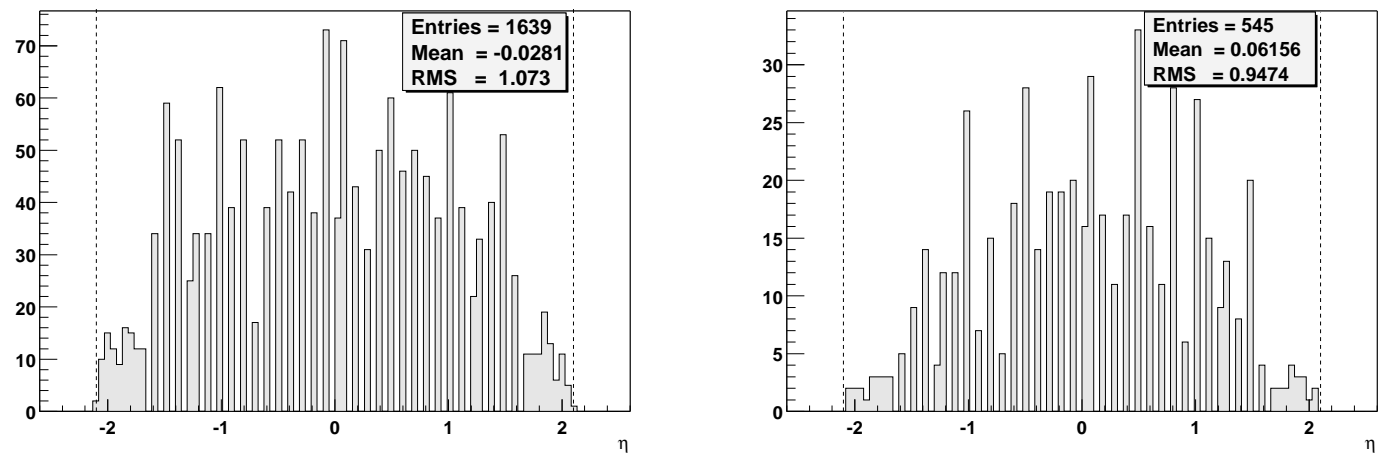

Figure 11: $\eta$-distributions of the two highest- $p_{T} \mathrm{~L} 1$ muon candidates for the smuon channel A (left) and the gluino/squark channel C (right).

only $37 \%$ for the same cuts. If at Level-1 a three-jet trigger with a threshold of $86 \mathrm{GeV}$ for each jet and at the High Level Trigger a four-jet trigger with thresholds of $113 \mathrm{GeV}$ are used in addition, mSUGRA4 goes from $92 \%$ cumulative L1 efficiency to $69 \%$ combined efficiency, and mSUGRA6 from $85 \%$ to $44 \%$. The efficiencies cited here can of course be improved by adding more algorithms and optimizing the conditions for supersymmetry once LHC has been turned on.

\section{Conclusions}

The efficiencies to trigger on supersymmetric events with the CMS Level-1 Trigger have been studied. Calculations have been performed for the production of smuon pairs, charginos/neutralinos, gluinos/squarks and general mSUGRA events at selected strategic points in the SUSY parameter space. A WW control channel with the W's decaying muonically was also studied. The energy and momentum thresholds were chosen to yield a total Level-1 trigger rate of about $16 \mathrm{kHz}$ at a luminosity of $2 \times 10^{33} \mathrm{~cm}^{-2} \mathrm{~s}^{-1}$, allowing for a safety factor of three with respect to the maximum HLT input rate of $50 \mathrm{kHz}$.

The Level-1 efficiencies for the majority of the studied channels are found to be good. They are between $88 \%$ and $96 \%$ for the general mSUGRA channels, between $93 \%$ and $100 \%$ for the smuon and gluino/squark channels and $81 \%$ for the WW channel. The chargino/neutralino channel shows the lowest efficiency, about $72 \%$. It can be interpreted by the fact that a large fraction of the energy is carried away by invisible particles and that the net missing transverse energy of the events is balanced.

The final efficiencies to trigger on interesting events depend both on the Level-1 and the High Level Trigger. The HLT in particular has a large potential to optimize its conditions for the on-line selection of supersymmetric events.

\section{Acknowledgements}

We would like to thank the Austrian Exchange Service (Österreichischer Austauschdienst) and the Hungarian government for their financial support (contract numbers A-28/2000 and TéT A-28/2000 respectively). We are grateful for a grant by the City of Vienna's Jubilee Fund for the Austrian Academy of Sciences (Jubiläumsfonds der Stadt Wien für die Österreichische Akademie der Wissenschaften). We are also indebted to our CMS colleagues S. Abdullin, S. Arcelli, P. Chumney, D. Acosta, D. Newbold and the numerous physicists, engineers and technicians that have made contributions to simulation software, analysis tools and Monte Carlo productions as well as detector and trigger development. 


\section{References}

[1] The TriDAS Project - The Level-1 Trigger Technical Design Report, CERN/LHCC 2000-38 (2000)

[2] The TriDAS Project - Data Acquisition and High Level Trigger Technical Design Report, CERN/LHCC 2002-26 (2002)

[3] J. Lackey et al., CMS Note 1998/074 (1998), “CMS Calorimeter Level-1 Regional Trigger Conceptual Design"

[4] G. P. Heath, Nucl. Instrum. and Meth. A 461 (2001) 505

[5] F. Gasparini et al., Nucl. Instrum. and Meth. A 336 (1993) 91

[6] J. Hauser, Proceedings of the Fifth Workshop on Electronics for LHC Experiments, Snowmass, Co., USA, Sept. 1999, CERN/99-09, CERN/LHCC/99-33 (1999) 304

[7] J. Erö, Proceedings of the Fifth Workshop on Electronics for LHC Experiments, Snowmass, Co., USA, Sept. 1999, CERN/99-09, CERN/LHCC/99-33 (1999) 309

[8] D. Acosta et al., Nucl. Instrum. and Meth. A 496 (2003) 64;

D. Acosta et al., Proceedings of the Seventh Workshop on Electronics for LHC Experiments, Stockholm, Sweden, Sept. 2001, CERN/2001-005, CERN/LHCC/2001-034 (2001) 243;

D. Acosta et al., Proceedings of the Fifth Workshop on Electronics for LHC Experiments, Snowmass, Co., USA, Sept. 1999, CERN/99-09, CERN/LHCC/99-33 (1999) 318

[9] M. Andlinger et al., Nucl. Instrum. and Meth. A 370 (1996) 389

[10] H. Sakulin, M. Fierro, CMS Note 2001/003 (2001), "Studies of the Global Muon Trigger Performance"

[11] C.-.E. Wulz, Nucl. Instrum. and Meth. A 473 (2001) 231;

A. Taurok, H. Bergauer, M. Padrta, Nucl. Instrum. and Meth. A 473 (2001) 243

[12] B.C. Allanach et al., Eur. Phys. J. C25 (2002) 113, "The Snowmass Points and Slopes: Benchmarks for SUSY Searches"

[13] N. Ghodbane, H.-U. Martyn, hep-ph/0201233 (2002), "Compilation of SUSY particle spectra from Snowmass benchmarks models"

[14] S. Abel et al., hep-ph/0003154 (2000), "Report of the SUGRA Working Group for RunII of the Tevatron"

[15] H. Baer et al., Phys. Rev. D51 (1995) 1046;

H. Baer et al., Phys. Rev. D 52 (1995) 2746

[16] T. Sjöstrand et al., Comput. Phys. Commun. 135 (2001) 238

[17] S. Mrenna, Comput. Phys. Commun. 101 (1997) 232, "SPYTHIA, A supersymmetric extension of PYTHIA"

[18] H. Baer, F.E. Paige, S.D. Protopopescu, X. Tata hep-ph/0001086 (2000), "ISAJET 7.48: A Monte Carlo Event Generator for $p p, \bar{p} p$, and $e^{+} e^{-}$Interactions". Version 7.51 was used for this study.

[19] H. Lai et al., Eur. Phys. J. C12 (2000) 375

[20] http://cmsdoc.cern.ch/cmsim/cmsim.html; C. Charlot et al., CMS TN/93-63 (1993), "CMSIM-CMANA CMS Simulation Facilities"

[21] GEANT3 Detector Description and Simulation Tool, CERN program library long writeup W5013, release 15111999 (1999)

[22] CMS Object Oriented Reconstruction, http://cmsdoc.cern.ch/orca/ 
[23] The CMS Hadron Calorimeter Project - Technical Design Report, CERN/LHCC 97-31 (1997)

[24] C. Zeitnitz, T. A. Gabriel, Nucl. Instrum. and Meth. A 349 (1994) 106; http://wswww.physik.uni-mainz.de/zeitnitz/gcalor/gcalor_manual.ps

[25] V. V. Abramov et al., Nucl. Instrum. and Meth. A 457 (2001) 75

[26] The CMS Electromagnetic Calorimeter Project - Technical Design Report, CERN/LHCC 97-33 (1997)

[27] A. Gresele, T. Rovelli, CMS Note 1999/064 (1999), "Parametrization of B Field Effects in DTBX"

[28] R. Wilkinson, P. T. Cox, CMS Note 2001/013 (2001), "Simulating the Endcap Muon CSC System in ORCA"

[29] G. Bruno, M. Konecki, CMS Note 2001/012 (2001), "Simulation of the Baseline RPC Trigger System for CMS: Efficiency and Output Rates in Single Muon Topology"

[30] D. Acosta, M. Stoutimore, S. M. Wang, CMS Note 2001/033 (2001), "Simulated Performance of the CSC Track-Finder" 